\title{
A Comparison of Multiple Chemical Sensitivity with Other Hypersensitivity Illnesses Suggests Evidence and a Path to Answers
}

Laurie Dennison Busby

Unaffiliated, Chesterfield, Missouri.

\section{Abstract}

Throughout history, when doctors and scientists have encountered illnesses that were not clearly visible and didn't have an obvious explanation, those illnesses have often been dismissed as either not real or as psychological. Asthma was among the illnesses once thought to be psychological. In order for doctors and researchers to reach similar conclusions about multiple chemical sensitivity, they have had to overlook a great deal of valuable evidence including the signs and symptoms of patients as well as the commonalities this illness shares with other now well-recognized hypersensitivity illnesses. Researching the relationship between multiple chemical sensitivity and other hypersensitivity illnesses may help narrow down the potential mechanisms in this illness and bring answers to patients, which might ease the burden of having an illness that is once again unrecognized by the people patients are most dependent on for help. Key Words: Multiple chemical sensitivity-Multiple drug hypersensitivity-Nonallergic asthma.

(C) Busby 2017; Published by Mary Ann Liebert, Inc. This Open Access article is distributed under the terms of the Creative Commons Attribution Noncommercial License (http://creativecommons.org/licenses/by-nc/4.0/) which permits any noncommercial use, distribution, and reproduction in any medium, provided the original author(s) and the source are credited.

\section{Introduction}

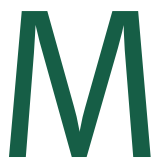

ultiple chemical sensitivity (MCS) and other hypersensitivity illnesses share signs and symptoms, similar triggers, a female predominance, and some test results. It has been known for a decade or more that some patients with MCS were found to have nasal pathology abnormalities (defects in tight junctions and "desquamation" of the respiratory epithelium); increased airway hyperresponsiveness to capsaicin, a TRPV1 agonist; increased plasma neuropeptides, which increased further after provocation; and xenobiotic-metabolizing enzyme (XME) polymorphisms (Kimata, 2004; Meggs, 1997; Millqvist, 2000; Schnakenberg et al., 2007). In the years since then, while vast strides have been made in uncovering some of the other mechanisms in well-recognized hypersensitivity illnesses such as asthma and chronic urticaria (CU), that same pace of advancement has not been made in MCS research. This is in part due to a lack of illness recognition despite the overlap between MCS and other hypersensitivity illnesses. Researching those relationships might bring answers and legitimacy to MCS. This review highlights some of the relevant past findings in MCS and other hypersensitivity conditions, their contribution thus far to the proof of MCS, and the need to focus on the findings in well-researched hypersensitivity illnesses in order to advance MCS research.

\section{MCS Signs and Symptoms}

In MCS, chronic fatigue syndrome (CFS) is often a comorbidity. CFS was found in $88.5 \%$ and $68.1 \%$ of non-occupational and occupational cases of MCS, respectively (Nogué Xarau et al., 2010). Patients with MCS or sometimes CFS may present with headaches; airway symptoms, especially upper airway symptoms; sometimes 


\section{COMPARISON: MCS AND OTHER HYPERSENSITIVITY ILLNESSES}

cutaneous symptoms; and/or adverse drug reactions to multiple medications (Baraniuk \& Zheng, 2010; Ferre Ybarz et al., 2005; Nijs et al., 2003; Ross, 1997; Ziem \& McTamney, 1997).

In patients with MCS and MCS-related disorders, "Preliminary data indicate the nasal pathology of these disorders is characterized by defects in tight junctions between cells, desquamation of the respiratory epithelium, glandular hyperplasia, lymphocytic infiltrates, and peripheral nerve fiber proliferation" (Meggs, 1997, p. 473). In CFS, there was a "high prevalence" of idiopathic nonallergic rhinopathy, and a subgroup of patients had bronchial hyperresponsiveness (Baraniuk \&t Zheng, 2010; Nijs et al., 2003).

Patients with MCS and CFS have reported drug reactions or drug "allergies" (Ferre Ybarz et al., 2005; Ross, 1997) Some of the reported drug "allergies" may actually have been drug reactions. IgEmediated drug allergies are "rare," especially to multiple medicines, and researchers noted total IgE was not highly elevated in most patients with MCS or CFS (Hasegawa et al., 2005; Kowal et al., 2002; Thalayasingam et al., 2013).

\section{Findings in Common with Other Hypersensitivity Illnesses \\ Similar triggers}

Airborne chemicals or irritants, even in natural fragrances, encountered in everyday life have been reported as triggers of symptoms in other hypersensitivity illnesses including asthma and migraine. "Air pollution is linked to increased emergency room visits for headache, and migraine patients frequently cite chemicals or odors as headache triggers" (Kunkler et al., 2015, p. 1192). "Odorants, ... especially perfume, may trigger migraine attacks after a few minutes of exposure" (Silva-Neto et al., 2014, p. 14). In asthmatics, after exposure to cologne, pulmonary function declined 18-58\% (Shim \&t Williams, 1986). This study concluded, "Odors are an important cause of worsening asthma" (Shim \&t Williams, 1986, p. 18).

\section{Gender prevalence}

In adults, there is a female predominance in MCS, CFS, and many of the hypersensitivity conditions including severe asthma, CU, multiple drug hypersensitivity (MDH), some types of angioedema (including acquired angioedema type II), and idiopathic anaphylaxis (Colombo et al., 2009; Moneret-Vautrin \& Gay, 1991; Nogué et al., 2007; Vohra et al., 2011; Zein et al., 2016).

\section{The immune system}

Based on what is known about other hypersensitivity conditions, it is likely that there are subgroups of and multiple mechanisms behind
MCS. Some patients with MCS or CFS have been found to have IgEmediated allergies; elevated inflammatory immune cells including Creactive protein; or an immune deficiency, which could contribute to respiratory infections and reactions (Hasegawa et al., 2005; Hilgers \& Frank, 1994; Kowal et al., 2002; Rea, 1979; Ziem \& McTamney, 1997). These were found in 10\%,10\%, and 15\% of patients with MCS, respectively (Rea, 1979). What about the rest of the patients?

In MCS and CFS, there is a female predominance (88\% in one MCS cohort), an increased frequency of Hashimoto's thyroiditis (HT)-related autoantibodies or positive anti-nuclear antibodies (ANA), and/or total IgE and eosinophils, which are not highly elevated in most patients (eosinophils were "depressed" in 90\% of patients with MCS) (Galland, 1987; Hilgers \&t Frank, 1994; Nogué et al., 2007; Rea, 1979; Ziem \&t McTamney, 1997). In addition, female gender and thyroid involvement have been associated with the propensity for having a reaction in MCS and CFS, respectively. The patients with MCS, who had trouble taking medicine, were more likely to be female (Suzuki et al., 2004). The subgroup of patients with CFS and bronchial hyperresponsiveness were more likely to have thyroid inflammation (Nijs et al., 2003). These findings suggest allergies are less likely and autoimmune mechanisms may be more likely to play at least a partial role in the hypersensitivity reactions in a subgroup of patients with MCS.

Within many of the hypersensitivity conditions, there are subgroups thought to have an autoimmune basis to their illness. These include many of the patients with nonallergic asthma, aka intrinsic asthma, and $\mathrm{MDH}$, and patients with chronic autoimmune urticaria. Patients within these autoimmune subgroups, despite having different conditions, may be more likely than their counterparts with the same hypersensitivity condition to share similarities, including a female predominance; an increased frequency of HT-related autoantibodies or positive ANA; a propensity toward other autoantibodies, that may contribute to their hypersensitivity reactions; a positive autologous serum skin test (ASST), a marker of self-reactivity to a patient's own serum; total IgE and eosinophils that are normal or even low; and/or more severe reactions than most patients with allergies.

The female predominance in some hypersensitivity illnesses may in part be due to an increased female prevalence within the autoimmune subgroups. In a cohort of nonallergic asthmatics, 65\% were female. When patients were further subgrouped based on test results thought to reflect potential autoimmunity, the female prevalence was even higher. Among those who were ASST-positive, 83\% were female (Comi et al., 2007). The ASST was negative in three control groups: allergic asthma, allergic rhinitis, and normals (Comi et al., 2007).

Some patients with hypersensitivity illnesses such as rhinitis, asthma, or CU, especially those with HT-related autoantibodies or 


\section{BUSBY}

ANA, may develop other autoantibodies that contribute to their reactions. In these patients, there is often a trend toward an inverse relationship between the frequency and/or levels of autoantibodies versus that of IgE and eosinophils.

In a cohort with chronic asthma and other lower respiratory illnesses (in which most also had sinusitis), autoantibodies to sinus and lung in sera were found in 46\% and 62\% of patients, respectively, but none of the healthy controls (Quintero et al., 1966). While this study did not make the following comparisons, this cohort seems to follow a trend similar to that in some cohorts with other hypersensitivity illnesses; the subgroup of patients with a low percentage of eosinophils was more likely to have an increased frequency of autoantibodies. When patients in this cohort are subgrouped by those with a low versus high percentage of eosinophils (4\% or less vs. $11 \%$ or more), 90\% versus $43 \%$ had autoantibodies, $40 \%$ versus $14.3 \%$ had autoantibodies to sinus and lung, and 50\% versus $28.6 \%$ had autoantibodies to sinus or lung, respectively. (Two patients with bronchiectasis and one patient with cystic fibrosis with low eosinophils were included in these figures since excluding them would not have changed these results significantly. Patients with emphysema tended to have both high eosinophil percentages [4/6] and an increased frequency of autoantibodies [6/6], and they were not included in these figures.)

In another asthma cohort (in which 82\% also reported rhinitis), compared with allergic asthma, females with nonallergic asthma had an increased frequency of autoantibodies to cytokeratin (CK), a bronchial epithelial antigen (40\% vs. 12\%); higher levels of anti-CK; and higher levels of anti-thyroid peroxidase, despite excluding patients with definite thyroid disease (Mohammad et al., 2015). There was a negative association between thyroid peroxidase autoantibody levels and total IgE (Mohammad et al., 2015).

Autoantibodies to beta 2 adrenergic receptors were also found in approximately $40 \%$ of asthmatics compared with approximately $5 \%$ of normal controls (Turki \& Liggett, 1995). Although it is unclear what role beta adrenergic receptor autoantibodies may play in CFS, these autoantibodies along with autoantibodies to muscarinic cholinergic receptors were found in $29.5 \%$ of patients with CFS. In addition, patients with CFS versus healthy controls had significantly higher levels of beta 2 adrenergic receptor autoantibodies, and patients with higher levels also more frequently had anti-thyroid peroxidase or ANA (Loebel et al., 2015). The frequency of these autoantibodies might be even higher in a CFS subgroup with rhinitis and/or bronchial hyperresponsiveness or in a CFS cohort of newly diagnosed untreated patients. This study excluded patients on steroids and other immunosuppressants, which also likely excluded some patients with autoimmune diseases, possibly patients who had had these autoantibodies.

The frequency of autoantibodies to tubulin was increased in a cohort with allergic rhinitis (52\%) (vs. none of the healthy controls); in a cohort with HT (56\%) and Grave's disease (41\%) (vs. patients with lupus [5\%], pernicious anemia [6\%], and toxic adenoma [1/11]); and in a cohort with infectious mononucleosis $14 \%$ (vs. patients with other viruses [7\%] and healthy lab workers [3\%]) (Mead et al., 1980; Nakamura et al., 2004; Rousset et al., 1983). In keeping with previous research findings, in anti-tubulin-positive versus anti-tubulinnegative patients from the first cohort, the female ratio was higher (F:M 8:5 vs. 4:8, respectively); and the RAST titers were lower, although this was not considered significant (cedar 12.4 [+- 24.6] vs. 22.3 [+- 32.4], house dust 33.1 [+- 42.2] vs. 60.6 [+- 75.1], mite 11.9 [+- 18.6] vs. 19.7 [+- 28.3], and ragweed/hogweed 9.1 [+- 11.4] vs. 24.9 [+- 32.4]) (Nakamura et al., 2004).

Interestingly, in a study that tested for autoantibodies including anti-tubulin during lupus exacerbations and remission, autoantibodies were found in the majority of samples taken during exacerbations but in very few samples taken during remission (Pateraki et al., 1986). This might account for some of the discrepancies between studies and has implications for the timing of testing in the future.

Autoantibodies can sometimes be found at low levels in healthy controls. Importantly, when IgM autoantibodies to tubulin were also tested, of the autoantibody positive patients, $72 \%$ of patients with infectious mononucleosis had high levels, while $72 \%$ of the patients with other viruses and healthy controls had only low levels (Mead et al., 1980).

While the autoantibodies were to beta-tubulin isoform $\mathrm{V}$ in the cohort with allergic rhinitis, the specific tubulin isoform and whether the patients also had respiratory symptoms is unknown in the latter two cohorts. However, the findings of tubulin autoantibodies in all three cohorts is interesting since the subgroup of patients with CFS, bronchial hyperresponsiveness, and thyroid inflammation was also more likely to have recurrent flu-like illness and painful lymph nodes (Nijs et al., 2003).

Environmental exposures (chemical or infectious) have been associated with the development of autoantibodies, and viruses are suspected triggers in many of the autoimmune diseases. It is possible a virus may have triggered autoantibody production in some patients with CFS. In addition, an increased frequency of autoantibodies to CK has been found in chemical-induced asthma from toluene diisocyanate (TDI) versus allergic asthma, TDI-exposed asymptomatic individuals, and healthy controls (Ye et al., 2006). Autoantibody levels can also increase with duration of an illness (Meszaros et al., 2010). 


\section{COMPARISON: MCS AND OTHER HYPERSENSITIVITY ILLNESSES}

This might be one potential pathway contributing to the "spreading phenomena" in some patients with MCS.

As mentioned earlier, patients that fall within the autoimmune subgroups may be less likely to have increased total IgE and IgEmediated histamine release. Instead, they may be more likely to have a positive ASST and high-affinity IgE receptor (FcepsilonRI aka FceRI) autoantibodies or other factors, which could cause histamine release from basophils and anaphylaxis in some patients (Marone et al., 1999). In a cohort of patients who had had drug reactions to one or more antibiotics, patients with MDH seldom had IgE to specific medications but were more than twice as likely to have a positive ASST, while single drug reactors were more likely to have drugspecific IgE but less likely to have a positive ASST (Asero et al., 2003). Of the patients with MDH versus single drug reactors, IgE to betalactams was found in 9\% (1 patient) versus 36\% of patients, respectively (Asero et al., 2003). None of the normal controls had a positive ASST (Asero et al., 2003).

Approximately $30-50 \%$ of patients with CU may have autoantibodies to FcepsilonRI or much less frequently to IgE itself, and these patients are considered to represent an autoimmune subgroup (Greaves, 2003). In one study, anti-FcepsilonRI were found in 37\% of patients with CU and none of the patients with atopic dermatitis or healthy controls (Fiebiger et al., 1995). Patients with CU and HTrelated autoantibodies were found to have anti-FcepsilonRI and even sera without anti-FcepsilonRI capable of causing histamine release from basophils (Kandeel et al., 2001). Interestingly, anti-FcepsilonRI were found in approximately 35\% of patients with CU, who reported natural aromas and food additives triggered their symptoms (Zuberbier et al., 2000).

In asthmatics, 29.5\% were thought to have anti-FcepsilonRI, and $32 \%$ had a positive basophil histamine release assay versus $9.4 \%$ and 9.4\% in healthy controls (Sun et al., 2008).

Patients within these autoimmune subgroups may also have move severe or extensive reactions. ASST-positive versus ASST-negative patients with asthma had a significantly increased airway hyperresponsiveness to methacholine challenge (Jang et al., 2007). An association was also found between anti-thyroid peroxidase levels and the severity of bronchial asthma (Mohammad et al., 2015). ASSTpositive versus ASST-negative patients with CU were more likely to have a higher mean urticaria activity score, respiratory or gastrointestinal symptoms, and throat angioedema (Vohra et al., 2011). Patients with CU and autoimmune thyroid disease had a 16 times greater risk of angioedema (Missaka et al., 2012).

The basophil activation test (BAT) can measure in vitro basophil reactivity to chemicals and could be used in patients with MCS. Pa- tients with autoimmune urticaria and a positive BAT were more likely to have autoimmune diseases, anti-thyroid antibodies, and higher total urticaria scores (Irinyi et al., 2007).

Besides B cells, $\mathrm{T}$ cells may play a role in some patients. $\mathrm{T}$ cell activation and/or a decrease in regulatory $\mathrm{T}$ (Treg) cells have also been associated with reactions to medicines. The lymphocyte transformation test (LTT) can measure in vitro lymphocyte reactivity to chemicals and metals and could be used in patients with MCS. The LTT has been used previously in MCS (Pigatto et al., 2013).

In patients with MDH and a positive in vitro LTT to more than one drug versus monoallergic patients, the drug-reactive $\mathrm{T}$ cells were found in a "pre-activated T cell fraction", which might result in a "lower threshold for activation by drugs" (Daubner et al., 2012, p. 58). The authors point out herpes viruses induce a similar T cell phenotype (Daubner et al., 2012). Interestingly, in a study of a patient with drug hypersensitivity and concurrent human herpes virus (HHV) 6 and HHV 7, "Drug-specific lymphocytes could be detected by LTT when the HHV was active (positive PCR for viral DNA and increased antiHHV $6 \operatorname{lgG}$ titer), but not when it was no longer active" (Calligaris et al., 2009, p. 173). This may have implications for patients who have virally triggered CFS and MCS.

\section{The epithelial barrier and defects in tight junctions}

The epithelial barrier helps protect the airways from inhaled irritants and infectious agents, and defects in the tight junctions allow inhaled substances increased access to airways. This may be more likely to trigger an immune response (Xiao et al., 2011, p. 549). This might also increase the unpleasant effects evoked by airborne chemicals and irritants.

Exposure to chemicals have been shown to result in "disorganized tight and gap junctions" (Lange et al., 1999). People with respiratory illnesses may be more sensitive to these effects. Asthmatics were found to have defects in tight junctions and cultures "more sensitive to disruption by cigarette smoke extract” (Xiao et al., 2011, p. 549).

While tight junctions and other junctions are maintained through multiple pathways including claudin, occludin, and cadherin, conversely, proteinases aka proteases are enzymes involved in junction degradation. Protease inhibitors alpha-1 antitrypsin and C1 inhibitor keep proteases in check and help maintain the epithelial and endothelial barriers. When $\mathrm{C} 1$ inhibitor is low, bradykinin (BK) increases and vascular endothelial (VE)-cadherin decreases, and this "causes the glue between the cells to disappear and causing the cells to centripetally contract" (Zuraw, 2010, p. 21).

In exhaled breath condensate, nonatopic and atopic asthmatics versus controls were found to have decreased epithelial (E)-cadherin: 


\section{BUSBY}

.106 (.089) and .112 (.060) versus .191 (.184) ng $\mathrm{mL}$, respectively (Yuksel et al., 2014). A C1 inhibitor deficiency and resulting increase in BK and decrease in VE-cadherin could also contribute to respiratory symptoms and angioedema including potentially life-threatening laryngeal edema (Bork et al., 2012).

A C1 inhibitor deficiency was found in a cohort with CFS (Hilgers Et Frank, 1994). In addition to defects in tight junctions and "desquamation" of the respiratory epithelium, throat discomfort (soreness, tightness) was found in 53\% of patients with MCS (Meggs, 1997; Ziem \& McTamney, 1997). It is possible a deficiency of proteins involved in junction maintenance or protease inhibitors, such as the $\mathrm{C} 1$ inhibitor, that protect them from degradation might play a role in the symptoms of some of the patients with MCS.

\section{Neurogenic inflammation}

Bradykinin along with nerve growth factor (NGF), substance $\mathrm{P}$ (SP), and vasoactive intestinal peptide (VIP) are mediators that can lead to neurogenic inflammation in sensory neurons. An increase in neuropeptides and a decrease in neuropeptide-degrading enzymes in nasal mucosa play a role in chronic inflammatory rhinitis and its severity (Lacroix, 2003; Landis et al., 2008).

A potential relationship between neurogenic inflammation and MCS was first suggested over 20 years ago (Meggs, 1993). Since then, in one MCS cohort, plasma NGF, SP, and VIP were found to be increased prior to provocation with volatile organic compounds (VOCs), and after provocation they were increased further along with an increase in skin wheel responses to histamine (Kimata, 2004). "These results indicate that exposure to VOC may enhance neurogenic inflammation with concomitant enhancement of histamine-induced responses" (Kimata, 2004, p. 159). Exposure to VOCs did not have these effects in normal subjects (Kimata, 2004).

One way BK and/or NGF can excite and sensitize sensory nerves is by activating transient receptor potential A1 and V1 (TRPA1 and TRPV1), known as the irritant receptor and capsaicin receptor, respectively.

\section{TRPA1 and TRPV 1}

"TRPA1 is expressed in sensory nerves and mediates cold, mechanical, and chemical nociception" (Yu \& Ouyang, 2009, para. 3). It is "capable of initiating reflex responses to many reactive chemical stimuli” (Taylor-Clark et al., 2009, p. 756).

TRPA1 and/or TRPV1 can be activated by airborne or ingested chemicals or irritants: anesthesia, wood smoke, and even the naturally occurring chemicals in pungent spices (Cornett et al., 2008; Iwasaki et al., 2008; Shapiro et al., 2013). While general anesthetics are considered central nervous system depressants, many of them actually stimulate peripheral sensory nerves by activating TRPA1 and TRPV1 (Cornett et al., 2008). This might help explain how things generally thought of as making people less reactive to stimuli, such as anesthesia, or things considered fairly innocuous, such as pungent spices, could trigger symptoms in some people.

TRPA1 and/or TRPV1 may play a role in the hypersensitivity illnesses in which odors trigger symptoms. "TRPA1 is emerging as a major contributing pathway in migraine" and "TRPA1 is also implicated in the pathogenesis of asthma" (Benemei et al., 2014, p. 2552; Deering-Rice et al., 2015, p. 893). TRPV1-like immunoreactive innervention was increased in the scalp arteries of migraineurs, and TRPV1 expression was increased in nasal mucosa in idiopathic rhinitis (Del Fiacco et al., 2015; Van Gerven et al., 2014). In some children with asthma, gene polymorphisms for TRPA1 were associated with reduced asthma control (Deering-Rice et al., 2015).

TRPA1 and/or TRPV1 may also play a role in MCS and CFS. When compared with asthmatics, patients with an MCS-like illness (sensitivity to chemical irritants, asthma-like symptoms, and no IgEmediated allergy) were more sensitive to inhaled capsaicin, a TRPV1 agonist (Millqvist, 2000). Patients with CFS compared to controls had a greater increase in TRPV1 mRNA after exercise (White et al., 2012).

Interestingly, insects and other animals exposed to chemicals, even naturally occurring chemicals, which activate TRPA1, avoid those substances. "Sensing and responding to changes in the external environment is important for insect survival ... pungent natural compounds ... activated Harm TRPA1 (a Helicoverpa armigera moth TRPA1 gene) ... Harm TRPA1 may function as ... a chemical sensor" (Wei et al., 2015, p. 412).

Wild-type (WT) mice would not enter a chamber filled with vapor of formalin (which contains formaldehyde), allyl isothiocyanate, and acrolein, while TRPA1 knockout (KO) mice entered the chamber "without hesitation" (Yonemitsu et al., 2013). In addition, formalin vapor woke WT mice from sleep but not TRPA1 K0 mice (Yonemitsu et al., 2013). The authors concluded, "Our results indicate that WT mice detect and recognize formalin, allyl isothiocyanate, and acrolein as dangerous substances via TRPA 1 ... stimulation of TRPA1 induces active avoidance behaviors, in addition to respiratory arrest (passive avoidance behavior) and pain sensitization" (Yonemitsu et al., 2013, Discussion section para. 1).

Because of their ability to modify responses in sensory nerves to airborne and ingested chemicals, this has led to the suggestion that TRPA1 antagonists or agonists may have "novel" uses in treating migraines and in pest control (Benemei et al., 2014; Wei et al., 2015). 


\section{COMPARISON: MCS AND OTHER HYPERSENSITIVITY ILLNESSES}

\section{Xenobiotic-metabolizing enzymes}

Some XME gene polymorphisms and/or altered levels/activity are seen in MCS and CFS (Caccamo et al., 2013; Schnakenberg et al., 2007). It is well known XME polymorphisms play a role in drug reactions, and they are under investigation in asthma and chemicalinduced asthma as well. XMEs are found in the olfactory epithelia and bronchiolar epithelium. "In addition to protecting against inhaled toxic compounds, these enzymes could also metabolize odorant molecules, and thus modify their stimulating properties or inactivate them" (Thiebaud et al., 2013, p. e59547). Inhibitors of these enzymes increased the electro-olfactogram response, "likely due to enhanced olfactory sensory neuron activation in response to odorant accumulation" (Thiebaud et al., 2013, p. e59547).

Olfactory mucosa metabolism for some compounds can be 3- to 65-fold higher than hepatic (Thiebaud et al., 2013). If the body has a greater need for XME activity in the olfactory mucosa, possibly as a first line of defense from airborne chemicals that might enter the central nervous system, then lower levels or activity of these enzymes may expose patients to higher levels or longer effects of airborne chemicals.

\section{Potential mediators and more systemic reactions}

Provocation tests of patients with MCS and patients with rhinitis have shown activation of mediators in nasal mucosa and plasma (Kimata, 2004; Millqvist et al., 2005). Potential mediators may not only alter the threshold of sensory neurons to excitation and sensitization, but they may also contribute to the increase in heart rate and some of the other central nervous symptoms. While IgE-mediated histamine release is unlikely to be responsible for the hypersensitivity reactions in most cases of MCS or CFS, regardless of the mechanism, "near" 40\% of patients from a cohort of patients with electrohypersensitivity and/or MCS had increased histamine (Belpomme et al., 2015). "Histamine has been known as a cardiac stimulant for over 70 years," was the first sentence of a paper written over 30 years ago, which makes this a fact that has been known for over a century (McNeill, 1984, p. 720). In addition, an intravenous injection of the TRPA1 agonists allyl isothiocyanate (found in mustard) and cinnamaldehyde (found in cinnamon) increased adrenaline secretion in lab animals (Iwasaki et al., 2008).

Nasally administered challenges can activate spinal trigeminal nerves. "Additionally, the spinal trigeminal nucleus, the first relay neurons of the trigeminal system, showed massive expression of cFos after a brief ( $3 \mathrm{~min}$ ) exposure to formalin vapor" (Yonemitsu et al., 2013, p. 3100).

Patients with MCS, like patients with CFS or possibly rhinitis, may have underlying autonomic dysfunction, making them more vul- nerable to these effects. Some patients with CFS have increased heart rate and reduced heart rate variability that persist during sleep accompanied by a trend toward increased norepinephrine (Boneva et al., 2007). In addition,

Thirty patients with perennial vasomotor rhinitis were submitted to pharmacological tests to access the autonomic responsiveness of extra-nasal and intra-nasal receptors to isoprenaline ... Isoprenaline induced a similar tachycardia in all patients, more pronounced than that observed in normal subjects ... Mequitazine improved significantly the clinical symptoms but did not modify the autonomic reactivity. (Mayeux et al., 1986, p. 330)

\section{Conclusion}

Multiple chemical sensitivity and other hypersensitivity illnesses share signs and symptoms, similar triggers, a female predominance, and some test results: defects in tight junctions, increased neuropeptides after provocation, XME polymorphisms, and probably TRPV1 activation. One way to expedite MCS research in the future is to focus on other applicable research findings from well-known hypersensitivity illnesses.

Based on the findings in those other illnesses, it is likely there are subgroups of patients with MCS. Testing for the autoantibodies seen in other respiratory illnesses might be productive in a potential autoimmune subgroup of patients with MCS, especially female patients with respiratory symptoms and HT-related autoantibodies or ANA. Among the other tests that may be useful in MCS are included the BAT and LTT, which can measure in vitro reactivity to chemicals.

As for the limitations of this review, there was not enough time to discuss all the possibly pertinent research on other hypersensitivity illnesses, and there was a paucity of research on MCS with which to compare it. Many of the markers found in other hypersensitivity illnesses have not yet been tested in MCS except in individual patients by their doctors. In addition, often in MCS research and sometimes in CFS research, when markers have been tested, only one team has tested them, making it difficult to say for certain whether they represent most patients or just that cohort due to the methods that were used, including exclusionary criteria.

In CFS research, thyroid disease or other autoimmune diseases have sometimes been among the exclusionary criteria, once again making it difficult to make comparisons. Several MCS researchers also consider autoantibodies seen in other respiratory illnesses exclusionary criteria (personal conversations). In diseases with a female predominance and in which so little is known, excluding those 


\section{BUSBY}

patients may exclude some of the sicker patients with MCS if it follows the patterns seen in other hypersensitivity illnesses.

This review also did not have the space to include fibromyalgia, another frequent comorbidity in MCS. In fibromyalgia, some markers have been found that might help explain MCS in those patients.

As discussed above, other hypersensitivity illnesses and MCS share signs and symptoms, similar triggers, a female predominance, and several research findings. These clues and this potential evidence should have been enough to spur vast strides in MCS research over the same time period in which similar findings spurred research advances in well-recognized hypersensitivity conditions. However, that hasn't happened, and neither has illness recognition. Meanwhile, patients with MCS wait, sometimes decades, while likely living with markers seen in other hypersensitivity illnesses that would be taken seriously if doctors or researchers were willing to test for them.

While MCS is currently not recognized by mainstream medicine, based on the commonalities it shares with other hypersensitivity illnesses, there is no doubt that it will be someday. When that time comes, the doctors and researchers who continued to dismiss patients with MCS will likely be viewed through the same lens we now use to view the doctors who at one time dismissed patients with asthma.

The famous author Marcel Proust (1871-1922) suffered from asthma during a time when it was considered psychological (Bogousslavsky, 2007). Like patients with MCS, Proust likely yearned for doctors to open their eyes and recognize how truly sick he and other patients with asthma were. That didn't happen in his lifetime.

Patients with MCS deserve to receive answers in their lifetime. Hopefully, this paper will open enough eyes to get them closer to that goal. After all, "The real voyage of discovery consists not in seeking new landscapes but in having new eyes" (Proust).

\section{Author Disclosure Statement}

No competing financial interests exist.

\section{REFERENCES}

Asero, R., Tedeschi, A., Lorini, M., Caldironi, G., \& Barocci, F. (2003). Sera from patients with multiple drug allergy syndrome contain circulating histaminereleasing factors. International Archives of Allergy and Immunology, 131, 195200.

Baraniuk, J. N., \& Zheng, Y. (2010). Relationships among rhinitis, fibromyalgia, and chronic fatigue. Allergy and Asthma Proceedings, 31, 169-178.

Belpomme, D., Campagnac, C., \& Irigaray, P. (2015). Reliable disease biomarkers characterizing and identifying electrohypersensitivity and multiple chemical sensitivity as two etiopathogenic aspects of a unique pathological disorder. Reviews on Environmental Health, 30, 251-271.
Benemei, S., Fusi, C., Trevisan, G. M., \& Geppetti, P. (2014). The TRPA1 channel in migraine mechanism and treatment. British Journal of Pharmacology, 171, 2552-2567.

Bogousslavsky, J. (2007). Marcel Proust's diseases and doctors: The neurological story of a life. Frontiers of Neurology and Neuroscience, 22, 89-104.

Boneva, R. S., Decker, M. J., Maloney, E. M., Lin, J. M., Jones, J. F., Helgason, H. G. ... Reeves, W. C. (2007). Higher rate and reduced heart rate variability persist during sleep in chronic fatigue syndrome: A population-based study. Autonomic Neuroscience, 137, 94-101.

Bork, K., Hardt, J., \& Witzke, G. (2012). Fatal laryngeal attacks and mortality in hereditary angioedema due to C1-INH deficiency. Journal of Allergy and Clinical Immunology, 130, 692-697.

Caccamo, D., Cesareo, E., Mariani, S., Raskovic, D., lentile, R., Curro, M. ... DeLuca, C. (2013). Xenobiotic sensor- and metabolism-related gene variants in environmental sensitivity-related illnesses: A survey on the Italian population. Oxidative Medicine and Cell Longevity, 2013, doi:10.1155/2013/831969

Calligaris, L., Stocco, G., De ludicibus, S., Marino, S., Decorti, G., Barbi, E., ... Ventura, A. (2009). Carbamazepine hypersensitivity syndrome triggered by a human herpes virus reactivation in a genetically predisposed patient. International Archives of Allergy Immunology, 149, 173-177.

Colombo, G., Yacoub, M. R., Burastero, S. E., Garattini, E., Girlanda, S., Saporiti, N., ... Sabbadini, M. G. (2009). Multiple drug hypersensitivity: Insight into the underlying mechanism and correlation with autoimmune diseases. European Annals of Allergy and Clinical Immunology, 41, 50-55.

Comi, A. L., Tedeschi, A., Lorini, M., \& Miadonna, A. (2007). Novel clinical and serological aspects in non-allergic asthma. Respiratory Medicine, 101, 2526-2533.

Cornett, P. M., Matta, J. A., \&t Ahern, G. P. (2008). General anesthetics sensitize the capsaicin receptor transient receptor potential V1. Molecular Pharmacology, 74, 1261-1268.

Daubner, B., Groux-Keller, M., Hausmann, O. V., Kawabata, T., Naisbitt, D. J., Park, B. K., ... Pichler, W. J. (2012). Multiple drug hypersensitivity: Normal Treg cell function but enhanced in vivo activation of drug-specific T cells. Allergy, 67, 58-66.

Deering-Rice, C. E., Shapiro, D., Romero, E. G., Stockmann, C., Bevans, T. S., Phan, 0. M., ... Reilly, C. A. (2015). Activation of transient receptor potential ankyrin-1 by insoluble particulate material and association with asthma. American Journal of Respiratory Cell and Molecular Biology, 53, 893-901.

Del Fiacco, M., Quartu, M., Boi, M., Serra, M. P., Melis, T., Boccaletti, R., ... Cianchetti, C. (2015). TRPV1, CGRP and SP in scalp arteries or patients suffering from chronic migraine. Journal of Neurology, Neurosurgery, and Psychiatry, 86, 393-397.

Ferre Ybarz, L., Cardona Dahl, V., Cadahia Garcia, A., Ruiz, E., Vazquez, A., Fernandez de Sevilla, T., \& Alegre Martin, J. (2005). Prevalence of atopy in chronic fatigue syndrome. Allergologia et Immunopathologia, 33, 42-47.

Fiebiger, E., Maurer, D., Holub, H., Reininger, B., Hartmann, G., Woisetschlager, M., ... Stingl, G. (1995). Serum IgG autoantibodies directed against the alpha chain of Fc epsilon RI: A selective marker and pathogenetic factor for a distinct subset of chronic urticaria patients? Journal of Clinical Investigation, 96, 2606-2612.

Galland, L. (1987). Biochemical abnormalities in patients with multiple chemical sensitivities. Occupational Medicine, 2, 713-720.

Greaves, M. W. (2003). Chronic idiopathic urticaria. Current Opinion in Allergy and Clinical Immunology, 3, 363-368.

Hasegawa, M., Ohtomo, M., Mita, H., \& Akiyama, K. (2005). Clinical aspects of patients with MCS-from the standpoint of allergy. Arerugi, 54, 478-484. 


\section{COMPARISON: MCS AND OTHER HYPERSENSITIVITY ILLNESSES}

Hilgers, A., \&t Frank, J. (1994). Chronic fatigue syndrome: Immune dysfunction, role of pathogens and toxic agents and neurological and cardial changes. Wiener Medizinische Wochenschrift, 144, 399-406.

Irinyi, B., Szeles, G., Gyimesi, E., Tumpek, J., Heredi, E., Dimitrios, G., ... Szegedi, A. (2007). Clinical and laboratory examinations in the subgroups of chronic urticaria. International Archives of Allergy and Immunology, 144, 217-225.

Iwasaki, Y., Tanabe, M., Kobata, K., \& Watanabe, T. (2008). TRPA1 agonists-allyl isothiocyanate and cinnamaldehyde-induce adrenaline secretion. Bioscience, Biotechnology, and Biochemistry, 72, 2608-2614.

Jang, A. S., Park, J. S., Lee, J. H., Park, S. W., Kim, D. J., \& Park, C. S. (2007). Autologous serum skin test for autoantibodies is associated with airway hyperresponsiveness in patients with asthma. Respiration, 74, 293-296.

Kandeel, A. A., Zeid, M., Helm, T., Lillie, M. A., Donahue, E., \&t Ambrus, J. L., Jr. (2001). Evaluation of chronic urticaria in patients with Hashimoto's thyroiditis. Journal of Clinical Immunology, 21, 335-347.

Kimata, H. (2004). Effect of exposure to volatile organic compounds on plasma levels of neuropeptides, nerve growth factor and histamine in patients with self-reported multiple chemical sensitivity. International Journal of Hygiene and Environmental Health, 207, 159-163.

Kowal, K., Schacterele, R. S., Schur, P. H., Komaroff, A. L., \&t DuBuske, L. M. (2002). Prevalence of allergen-specific $\lg E$ among patients with chronic fatigue syndrome. Allergy and Asthma Proceedings, 23, 35-39.

Kunkler, P. E., Zhang, L., Pellman, J. J., Oxford, G. S., \& Hurley, J. H. (2015). Sensitization of the trigeminovascular system following environmental irritant exposure. Cephalalgia, 35, 1192-1201.

Lacroix, J. S. (2003). Chronic rhinosinusitis and neuropeptides. Swiss Medical Weekly, 133, 560-562.

Landis, B. N., Grouzmann, E., Monod, M., Busso, N., Petak, F., Spiliopoulos, A., ... Lacroix, J. S. (2008). Implication of dipeptidy/peptidase IV activity in human bronchial inflammation and in bronchoconstriction evaluated in anesthetized rabbits. Respiration, 75, 89-97.

Lange, R. W., Lantz, R. C., Stolz, D. B., Watkins, S. C., Sundareshan, P., Lemus, R., \&t Karol, M. H. (1999). Toluene diisocyanate colocalizes with tubulin on cilia of differentiated human airway epithelial cells. Toxicological Sciences, 50, 64-71.

Loebel, M., Grabowski, P., Hiedecke, H., Baur, S., Hanitsch, L. G., Wittke, K., ... Carmen, M. (2015). Antibodies to beta 2 adrenergic and muscarinic cholinergic receptors in patients with chronic fatigue syndrome. Brain, Behavior, and Immunity, pii:S0889-1591(15)30020-9, doi:10:1016/j.bbi.2015.09.013

Marone, G., Spadaro, G., Palumbo, C., \& Condorelli, G. (1999). The anti-IgE/antiFcepsilonRlalpha autoantibody network in allergic and autoimmune diseases. Clinical \& Experimental Allergy, 29, 17-27.

Mayeux, D., Le Van, D., Pitois, M., Rivasseau, J., Kohler, C., \&t Grilliat, J. P. (1986). Autonomic dysfunction in perennial vasomotor rhinitis and the effect of mequitazine. Current Medical Research and Opinion, 10, 330-338.

McNeill, J. H. (1984). Histamine and the heart. Canadian Journal of Physiology and Pharmacology, 62, 720-726.

Mead, G. M., Cowin, P., \& Whitehouse, J. M. (1980). Antitubulin antibody in healthy adults and patients with mononucleosis and its relationship to smooth muscle antibody (SMA). Clinical \& Experimental Immunology, 39, 328-336.

Meggs, W. J. (1993). Neurogenic inflammation and sensitivity to environmental chemicals. Environmental Health Perspectives, 101, 234-238.
Meggs, W. J. (1997). Hypothesis for induction and propagation of chemical sensitivity based on biopsy studies. Environmental Health Perspectives, 105(Suppl 2), 473-478.

Meszaros, T., Fust, G., Farkas, H., Jakab, L., Temesszentandrasi, G., Nagy, G., ... Varga, L. (2010). C1-inhibitor autoantibodies in SLE. Lupus, 19, 634-638.

Millqvist, E. (2000). Cough provocation with capsaicin is an objective way to test sensory hyperactivity in patients with asthma-like symptoms. Allergy, 55, 546-550.

Millqvist, E., Ternesten-Hasseus, E., Stahl, A., \&t Bende, M. (2005). Changes in levels of nerve growth factor in nasal secretions after capsaicin inhalation in patients with airway symptoms from scents and chemicals. Environmental Health Perspectives, 113, 849-852.

Missaka, R. F., Penatti, H. C., Silvares, M. R., Nogueira, C. R., \& Mazeto, G. M. (2012). Autoimmune thyroid disease as a risk factor for angioedema in patients with chronic idiopathic urticaria: A case-control study. Sao Paulo Medical Journal, 130, 294-298.

Mohammad, H. A., Abdelfattah, M. T., Ali, L. H., \& Morsl, Z. I. (2015). Association between anti-thyroid peroxidase and anti-cytokeratin 18 autoantibodies and bronchial asthma in women. Egyptian Journal of Chest Diseases and Tuberculosis, 65, 7-14.

Moneret-Vautrin, D. A., \&t Gay, G. (1991). The so-called "idiopathic" anaphlaxis: Allergic and pseudo-allergic reactions. Allergie et Immunologie (Paris), 23, 89-93.

Nakamura, M., Tsutsumi, K., Ooka, S., \& Kato, T. (2004). Identification of beta-tubulin isoform $\mathrm{V}$ as an autoantigen in allergic rhinitis by a proteomic approach. Microbiology and Immunology, 48, 427-434.

Nijs, J., De Becker, P., De Meirleir, K., Demanet, C., Vincken, W., Schuermans, D., \&t McGregor, N. (2003). Associations between bronchial hyperresponsiveness and immune cell parameters in patients with chronic fatigue syndrome. Chest, 123, 998-1007.

Nogué, S., Fernandez-Sola, J., Rovira, E., Montori, E., Fernandez-Huerta, J. M., \&t Munne, P. (2007). Multiple chemical sensitivity: Study of 52 cases. Medicina Clinica, 129, 96-98; quiz99.

Nogué Xarau, S., Alarcon Romay, M., Matinez Matinez, J. M., Delclos Clanchet, J., Rovira Prat, E., \&t Fernandez Sola, J. (2010). Multiple chemical sensitivity: Epidemiological, clinical and prognostic differences between occupational and non-occupational cases. Medicina Clinica, 135, 52-58.

Pateraki, E., Kaklamani, E., Kaklamanis, P., Portocalas, R., \& Aessopos, A. (1986). Autoantibodies in systemic lupus erythematosus and normal subjects. Clinical Rheumatology, 5, 338-345.

Pigatto, P. D., Minoia, C., Ronchi, A., Brambilla, L., Ferrucci, S. M., Spadari, F., ... Guzzi, G. (2013). Allergological and toxicological aspects in a multiple chemical sensitivity cohort. Oxidative Medicine and Cell Longevity, 2013, doi:10.1155/ 2013/356235

Quintero, J. M., Burrell, R. G., Cather, C. I. I., Wymer, B., \&t Baise, R. (1966). Detection of antibodies to sinus tissue in chronic bronchial asthma. Journal of Allergy, 37, 84-89.

Rea, W. (1979). The environmental aspects of ear, nose, and throat disease: Part II. J.C.E.O.R.L. \& Allergy, 41, 41-54.

Ross, G. H. (1997). Clinical characteristics of chemical sensitivity: An illustrative case history of asthma and MCS. Environmental Health Perspectives, 105(Supp/ 2), 437-441.

Rousset, B., Bernier-Valentin, F., Poncet, C., Orgiazzi, J., Madec, A. M., Monier, J. C., \&t Mornex, R. (1983). Anti-tubulin antibodies in autoimmune thyroid disorders. Clinical \&t Experimental Immunology, 52, 325-332. 


\section{BUSBY}

Schnakenberg, E., Fabing, K. R., Stanulla, M., Stobl, N., Lustig, M., Fabing, N., \&t Schloot, W. (2007). A cross-sectional study of self-reported chemical-related sensitivity is associated with gene variants of drug-metabolizing enzymes. Environmental Health, 6, doi:10.1186/1476-069X-6-6

Shapiro, D., Deering-Rice, C. E., Romero, E. G., Hughen, R. W., Light, A. R., Veranth, J. M., \& Reilly, C. A. (2013). Activation of transient receptor potential ankyrin-1 (TRPA 1$)$ in lung cells by wood smoke particulate material. Chemical Research in Toxicology, 26, 750-758.

Shim, C., \& Williams, M. H., Jr. (1986). Effect of odors in asthma. American Journal of Medicine, 80, 18-22.

Silva-Neto, R. P., Peres, M. F., \& Valenca, M. M. (2014). Odorant substances that trigger headaches in migraine patients. Cephalalgia, 34, 14-21.

Sun, R. S., Chen, X. H., Liu, R. Q., Cheng, T. M., Ran, X. Z., \&t Yang, T. (2008). Autoantibodies to the high affinity lgE receptor in patients with asthma. Asian Pacific Journal of Allergy Immunology, 26, 19-22.

Suzuki, J., Nikko, H., Kaiho, F., Yamaguchi, K., Wada, H., \& Suzuki, M. (2004). The problems of multiple-chemical sensitivity in patients using medicinal drugs. Yagugaku Zasshi, 124, 561-567.

Taylor-Clark, T. E., Kiros, F., Carr, M. J., \& McAlexander, M. A. (2009). Transient receptor potential ankyrin 1 mediates toluene diisocyanate-evoked respiratory irritation. American Journal of Respiratory Cell and Molecular Biology, 40, 756-762.

Thalayasingam, M., Davies, L. J., Llanora, G. V., Gerez, I. F., Van Bever, H. P., \&t Shek, L. P. (2013). Clinical characteristics and outcomes of patients undergoing drug provocation tests (DPTs). Annals Academy of Medicine Singapore, 42, 184-189.

Thiebaud, N., Veloso Da Silva, S., Jakob, I., Sicard, G., Chevalier, J., Menetrier, F., ... Le Bon, A.-M. (2013). Odorant metabolism catalyzed by olfactory mucosal enzymes influences peripheral olfactory responses in rats. PLoS One, 8, doi:10.1371/journal.pone.0059547

Turki, J., \& Liggett, S. B. (1995). Receptor-specific functional properties of beta 2adrenergic receptor autoantibodies in asthma. American Journal of Respiratory Cell and Molecular Biology, 12, 531-539.

Van Gerven, L., Alpizar, Y. A., Wouters, M. M., Hox, V., Hauben, E., Jorissen, M., ... Boeckxstaens, G. (2014). Capsaicin treatment reduces nasal hyperactivity and

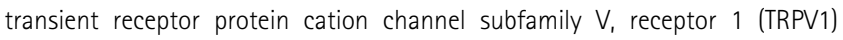
overexpression in patients with idiopathic rhinitis. Journal of Allergy and Clinical Immunology, 133, 1332-1339.

Vohra, S., Sharma, N. L., Mahajan, V. K., \& Shanker, V. (2011). Clinicoepidemiologic features of chronic urticaria in patients having positive versus negative autologous serum skin test: A study of 100 Indian patients. Indian Journal of Dermatology, Venereology and Leprology, 77, 156-159.

Wei, J. J., Fu, T., Yang, T., Liu, Y., \& Wang, G. R. (2015). A TRPA1 channel that senses thermal stimulus and irritating chemicals in Helicoverpa armigera. Insect Molecular Biology, 24, 412-421.
White, A. T., Light, A. R., Hughen, R. W., Vanhaitsma, T. A., \& Light, K. C. (2012). Differences in metabolite-detecting, adrenergic, and immune gene expression after moderate exercise in patients with chronic fatigue syndrome, patients with multiple sclerosis, and healthy controls. Psychosomatic Medicine, 74, 46-54.

Xiao, C., Puddicombe, S. M., Field, S., Haywood, J., Broughton-Head, V., Puxeddu, I., ... Davies, D. E. (2011). Asthma and lower airway disease: Defective epithelial barrier function in asthma. Journal of Allergy and Clinical Immunology, 128, 549-556.

Ye, Y. M., Nahm, D. H., Kim, C. W., Kim, H. R., Hong, C. S., Park, C. S., ... Park, H. S. (2006). Cytokeratin autoantibodies: Useful serologic markers for toluene diisocyanate-induced asthma. Yonsei Medical Journal, 47, 773-781.

Yonemitsu, T., Kuroki, C., Takahashi, N., Mori, Y., Kanmura, Y., Kashiwadani, H., ... Kuwaki, T. (2013). TRPA1 detects environmental chemicals and induces avoidance behavior and arousal from sleep. Scientific Reports, 3, doi:10.1038/srep03100

Yu, S., \& Ouyang, A. (2009). TRPA1 in bradykinin-induced mechanical hypersensitivity of vagal C fibers in guinea pig esophagus. American Journal of Physiology Gastrointestinal and Liver Physiology, 296, G255-G265.

Yuksel, H., Turkeli, A., Taneli, F., Dinc Horasan, G., Toprak Kanik, E., Kizilkaya, M., ... Yilmaz, O. (2014). E-cadherin as an epithelial barrier protein in exhaled breath condensate. Journal of Breath Research, 8, doi:10.1088/1752-7155/8/4/046006

Zein, J. G., Udeh, B. L., Teague, W. G., Koroukian, S. M., Schlitz, N. K., Bleecker, E. R., ... Erzurum, S. C. (2016). Impact of age and sex on outcomes and hospital cost of acute asthma in the United States, 2011-2012. PLoS One, 11, doi:10.1371/ journal.pone.0157301

Ziem, G., \& McTamney, J. (1997). Profile of patients with chemical injury and sensitivity. Environmental Health Perspectives, 105(Supp/ 2), 417-436.

Zuberbier, T., Henz, B. M., Fiebiger, E., Maurer, D., \& Stingl, G. (2000). Anti-FcepsilonRlalpha serum autoantibodies in different subtypes of urticaria. Allergy, 55, 951-954.

Zuraw, B. L. (2010). The pathology of hereditary angioedema. World Allergy Organization Journal, 3(Supp/ 3), doi:10.1186/1939-4551-3-S3-S25

Address correspondence to: Laurie Dennison Busby 1914 Claymills Dr. Chesterfield, MO 63017

E-mail: llbusby@charter.net

Received: January 9, 2017 Accepted: April 6, 2017 